\title{
A Closed Time-Reversal Cavity for Electromagnetic Waves in Transmission Line Networks
}

\author{
Zhaoyang Wang, Student Member, IEEE, Farhad Rachidi, Fellow, IEEE, Mario Paolone, Senior Member, IEEE, \\ Marcos Rubinstein, Fellow, IEEE, and Reza Razzaghi, Member, IEEE
}

\begin{abstract}
A closed time-reversal cavity has been considered a purely theoretical concept whose experimental realization was assumed to be impossible. In this paper, we demonstrate that it is, in fact, possible to realize a closed time-reversal cavity using a network of transmission lines. The network is excited by either lumped voltage or current sources at arbitrary locations, and it is terminated on matched impedances. This system is an exact closed time-reversal cavity in the sense that, in the backward-propagation stage, by back-injecting time-reversed measured responses (voltage or current) generated by a source at each line terminal, a time-reversed copy of the current and voltage distribution in the forward-propagation stage is obtained. We report for the first time an experimental realization of a time-reversal cavity formed by a network of inhomogeneous transmission lines, in which the spatial and temporal signal distribution along the network is reproduced as in a sequence of the system state (i.e., voltages and or current along the lines) being played in reverse. We also discuss the interfering effect of the so-called diverging wave for observation points in the vicinity of the source. An active realization of a time-reversal sink is proposed to effectively overcome the interfering effect caused by the diverging waves.
\end{abstract}

Index Terms-Electromagnetic time reversal, electromagnetic wave, time-reversal cavity, time-reversal sink, transmission line.

\section{INTRODUCTION}

$\mathbf{T}$ HE concept of a closed time-reversal cavity was proposed by D. Cassereau and M. Fink (e.g, [1]-[3]) for acoustic waves, and it was later extended to electromagnetic waves using the Lorentz reciprocity principle [4]. A time-reversal cavity extends the concept of a time-reversal mirror (e.g., [5][9]), a technique to refocus waves in a propagative medium back to the source [10]-[17].

A closed time-reversal cavity for electromagnetic waves can be briefly described as follows. Consider the situation wherein a source is situated in a linear, non-magnetic, and timeinvariant medium. Note that the medium can be inhomogeneous (see Fig. 1). Assume a closed, three-dimensional (3-D)

This work has been carried out within the frame of the Swiss Centre for Competence in Energy Research on Future Swiss Electrical Infrastructure (SCCER-FURIES) with the financial support of the Swiss Innovation Agency (Innosuisse-SCCER program) and the Swiss Federal Office of Energy (Contract SI/501706-01).

Z. Wang, M. Paolone, and F. Rachidi are with the Electromagnetic Compatibility Laboratory and the Distributed Electrical Systems Laboratory of the Swiss Federal Institute of Technology, Lausanne (EPFL), Switzerland (e-mail: zhaoyang.wang@epfl.ch, mario.paolone@epfl.ch, farhad.rachidi@epfl.ch).

M. Rubinstein is with the Institute for Information and Communication Technologies, University of Applied Sciences Western Switzerland, 1400 Yverdon-les-Bains, Switzerland. (e-mail: marcos.rubinstein@heigvd.ch).

R. Razzaghi is with the Department of Electrical and Computer Systems Engineering, Monash University, Australia (e-mail:reza.razzaghi@monash.edu). surface $S$ surrounding the source and its ambient medium [Fig. 1(a)]. The source emits an electromagnetic pulse. Suppose that we are able to determine the tangential fields generated by the source at any point on this surface [Fig. 1(b)]. Making use of the equivalence theorem, it is possible to replace the source by equivalent electric and magnetic current sources (on the surface $S$ ) [Fig. 1(c)]. Time reversing the equivalent sources on the surface results in the time reversal of the electromagnetic fields within the cavity surface [Fig. 1(d)]. An observer would see the fields propagate inwards, retracing the exact path the electromagnetic waves had followed as in a film being played in reverse or, equivalently, as if the observer were traveling backwards in time. The fields would then converge back to their originally-generated location.

The concept of closed time-reversal cavity allows therefore refocusing a wave back to its source, using a two-stage process. In the forward-propagation stage, generally named direct time (abbreviated as DT) in the time-reversal theory, the electromagnetic fields generated by the source are determined, either theoretically or experimentally, over the surface $S$ forming the cavity. Then in the backward-propagation stage, named reversed time (abbreviated as RT), the original source is removed from the medium and equivalent time-reversed sources (named secondary sources) on the surface are applied. The resulting field in the backward-propagation stage will be a time-reversed copy of that of the forward-propagation stage and, thus, it will converge back to the source.

It is generally assumed that the closed time-reversal cavity is impossible to achieve experimentally because it requires an infinite number of observation points (e.g., transducers) covering a closed 3-D surface around the medium to obtain information about all wavefronts propagating in all directions ${ }^{1}$ [1], [18]. In practice, the source-generated fields (e.g., acoustic or electromagnetic) are measured using a limited number of transducers in a layout over a finite angular aperture, namely a time-reversal mirror. Even though the focusing property of time reversal remains intact (e.g., [7], [18]-[20]), the field distribution resulting from the injection of the time-reversed fields back into the medium will not be an exact time-reversed copy of the fields in the forward stage.

In this paper, we demonstrate that a closed time-reversal cavity can, in fact, be realized using a transmission line net-

\footnotetext{
${ }^{1}$ One can use a finite number of transducers located at distances of $\lambda_{\min } / 2$, from one another, $\lambda_{\min }$ being the minimum wavelength associated with the source excitation. Still, the number of transducers can be quite significant for high-frequency sources, and the measured waveforms can be affected by cross-coupling between adjacent transducers.
} 


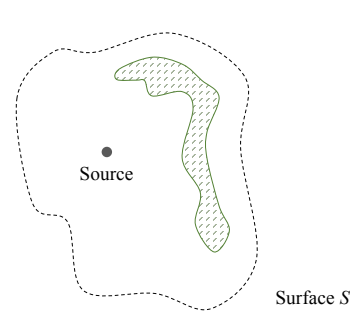

(a)

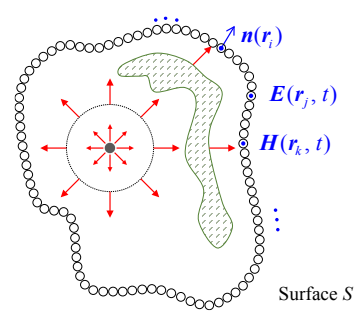

(b)

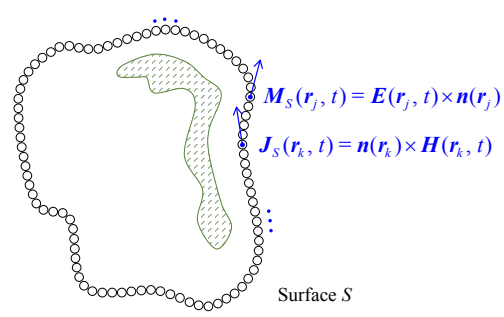

(c)

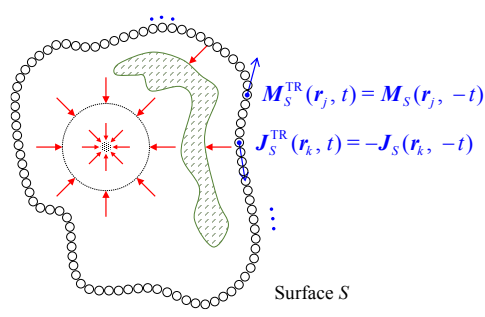

(d)

Fig. 1: Schematic description of a closed time-reversal cavity. (a) Assume a closed, three-dimensional surface $S$ surrounding a source and its ambient inhomogeneous medium. (b) The tangential fields generated by the source at any point on the surface $S$ are determined. (c) The source is replaced by equivalent electric and magnetic current sources $\boldsymbol{J}_{S}$ and $\boldsymbol{M}_{\boldsymbol{S}}$. (d) Time reversing the equivalent sources on the surface results in time reversing the electromagnetic fields within that surface.

work. Such a time-reversal cavity requires a finite number of observation points located at the network terminals.

This paper is structured as follows: Section II describes the realization of a closed time-reversal cavity for electromagnetic waves in transmission line networks. Section III illustrates the concept with a Y-shaped coaxial-cable network and it includes a derivation of the forward- and backward-prorogation stages' wave equations. In Section IV, a numerical and experimental validation of the proposed time-reversal cavity is presented. A discussion is given in Section $\mathrm{V}$ on the effects of the backwardstage diverging process and an active realization of a timereversal sink. Lastly, Section VI concludes the paper with final remarks.

\section{Realization of a Closed Time-Reversal CaVity USING A NETWORK OF TRANSMISSION LineS}

Consider a transmission line network formed by coaxial shielded cables with an arbitrary topology as shown in Fig. 2(a). Such networks can be found in various applications, e.g., in electronic and communications circuits, and in underground power networks. Let the transmission line network be excited by an impulsive voltage or current source located at an arbitrary point along one of the line branches [see the red dot along branch 1 in Fig. 2(b)]. Consider also a closed surface $S$ (dotted line in Fig. 2) that surrounds the network passing through each one of its terminals. Note that the network can be inhomogeneous and non-uniform in the sense that the transmission lines that form the network can have different characteristics, such as propagation speed and cross-sectional dimension. Note also that the network can have any number of branches and nodes, and any number of sources, either voltage or current.

Applying the concept of the closed time-reversal cavity to Fig. 2 implies considering a matched load at each line terminal in such a way that electromagnetic energy is only going outward from the cavity.

The electromagnetic field propagation in such system is confined within the cable (i.e., in the space between the inner conductor and the sheath). Furthermore, as long as the transverse dimensions of the cables forming the network are electrically small ${ }^{2}$, the wave propagation along the conducting cables is transverse electromagnetic (TEM) [21]. Therefore,

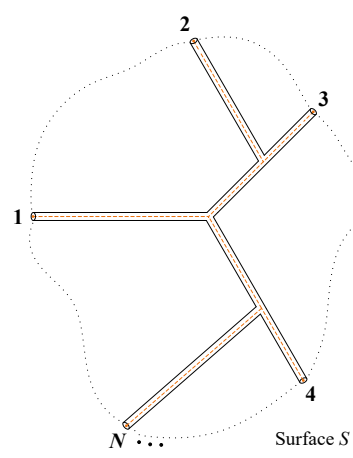

(a)

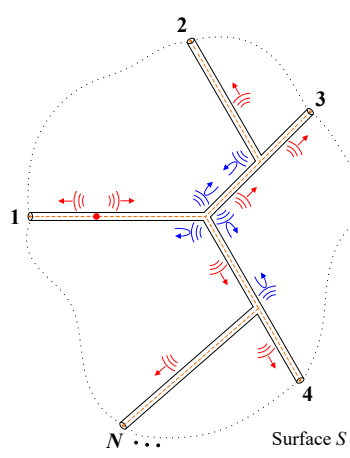

(b)
Fig. 2: A closed time-reversal cavity in a transmission line network composed of coaxial shielded cables. The network can have any arbitrary topology with any number of junctions and nodes. (a) A closed surface $S$ represented by a dotted line surrounds the network passing through each terminal of the network (labeled 1 to $N$ ). (b) The transmission line network is excited by a voltage or current pulse source (represented by the red dot). The transmitted electromagnetic waves (in red color) propagate along the network. At the inner nodes, parts of the waves are transmitted to connected branches and parts of them, shown in blue, are reflected back.

the field characteristics at every terminal are totally determined by the measured output signal (either current or voltage).

Furthermore, the only locations on the surface $S$ at which electromagnetic fields are nonzero are at the line terminals. In other words, the considered system allows realizing a closed time-reversal cavity using a finite number of measurements equal to the number of network terminals. The current or voltage distribution along the network, resulting from the back-injection of the time-reversed signals from all terminals, will be an exact time-reversed copy of its distribution in the forward-propagation stage.

\section{ILlustration of the CONCEPT OF A Closed TIME-REVERSAL CAVITY USING A Y-SHAPED TRANSMISSION LINE NETWORK}

\section{A. A closed time-reversal cavity in a Y-shaped transmission line network}

The proposed closed time-reversal cavity in a transmission line cable network is a general concept and it applies to any

\footnotetext{
${ }^{2}$ For example, a communication cable of about $1-\mathrm{cm}$ diameter is electrically small for signals with a frequency content of up to a few $\mathrm{GHz}$.
} 
arbitrary multi-terminal, multi-node topology. To illustrate the concept, we will consider here an inhomogeneous Y-shaped network (see Fig. 3), for which the solutions of the wave equations in the forward and the backward stages can be found analytically.

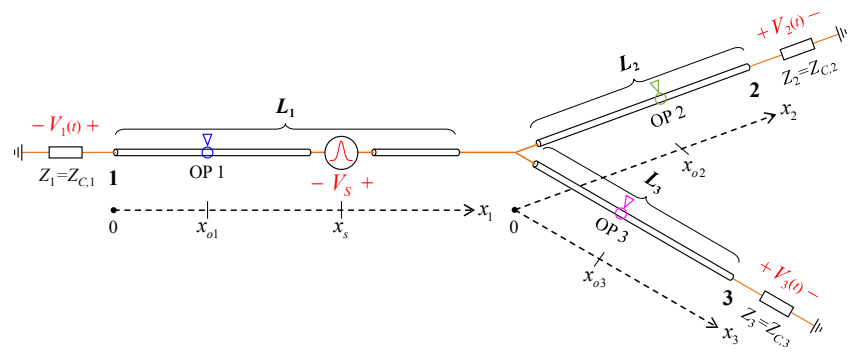

(a)

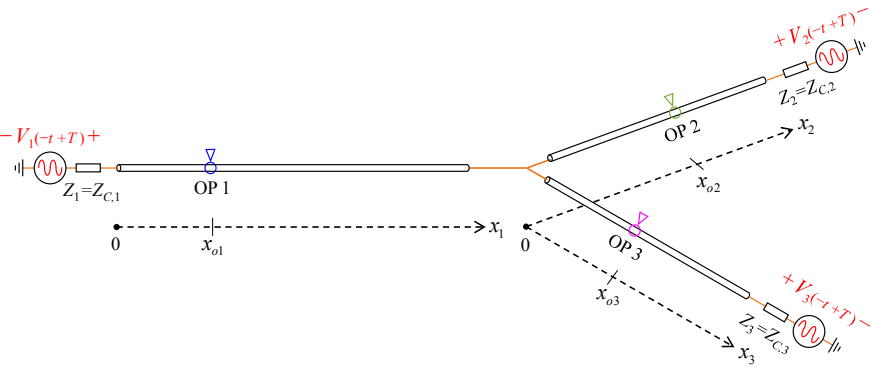

(b)

Fig. 3: Realization of a closed time-reversal cavity in a Y-shaped inhomogeneous coaxial-cable network. (a) Forward-propagation stage: direct time. (b) Backward-propagation stage: reversed time.

As illustrated in Fig. 3(a), in the forward-propagation stage, a voltage pulse is injected into the inner conductor of the coaxial cable at a given location along Line branch 1 (along the $x_{1}$ axis) and induced voltage responses are observed at the three network terminals (labeled 1 to 3 ). In the backwardpropagation stage [see Fig. 3(b)], the recorded voltages are time reversed and synchronously back injected into the network from the respective line terminals. In order to show that the temporal and spatial signal distribution along the entire network can be reproduced in reverse sequence, three arbitrary observation points (identified as OP 1-3 in Fig. 3) are considered.

\section{B. Wave equations in the closed time-reversal cavity}

In this section, wave equations are derived to describe the time-reversal forward- and backward-propagation processes in the Y-shaped cable network of Fig. 3. Without loss of generality, the derivation focuses on the voltage wave. To ensure the time-reversal invariance of the wave equations, let us assume for now a lossless medium.

We use voltage reflection coefficients in the transmission line theory to describe the boundary conditions at the network terminals and at the junction of the three line branches. Taking into account directionality, the instantaneous voltage reflection coefficient at the junction can be defined as

$$
\rho_{j, k}^{i}=\frac{Z_{j, k}-Z_{C, i}}{Z_{j, k}+Z_{C, i}}, i \in U=\{1,2,3\}, j, k=C_{U} i,
$$

where the superscript $i$ corresponds to the number of the line branch, along which incident voltage waves propagate, and the subscript pair $j, k$ combines the numbers of the other two lines connected to the junction. $Z_{C, i}$ refers to the line characteristic impedance and $Z_{j, k}$ is the input impedance of the other two line branches seen from the junction. The input impedance for a matched transmission line being equal to its characteristic impedance, $Z_{j, k}$ can be thus calculated as:

$$
Z_{j, k}=\frac{Z_{C, j} \cdot Z_{C, k}}{Z_{C, j}+Z_{C, k}} .
$$

In the forward-propagation stage [see Fig. 3(a)], responding to the series voltage source $V_{S}(t)$ exciting the coaxial-cable network at $x_{1}=x_{s}$, the voltage waves recorded at the terminals (representing the cavity surface) read:

$$
\begin{gathered}
V_{1}(t)=-\frac{1}{2} V_{S}\left(t-\frac{x_{s}}{v_{1}}\right)+\rho_{2,3}^{1} \cdot \frac{1}{2} V_{S}\left(t-\frac{L_{1}-x_{s}}{v_{1}}-\frac{L_{1}}{v_{1}}\right), \\
V_{2}(t)=\left(1+\rho_{2,3}^{1}\right) \cdot \frac{1}{2} V_{S}\left(t-\frac{L_{1}-x_{s}}{v_{1}}-\frac{L_{2}}{v_{2}}\right), \\
V_{3}(t)=\left(1+\rho_{2,3}^{1}\right) \cdot \frac{1}{2} V_{S}\left(t-\frac{L_{1}-x_{s}}{v_{1}}-\frac{L_{3}}{v_{3}}\right),
\end{gathered}
$$

which are obtained considering the matched boundary conditions at the network terminals. $v_{i}$ refers to the wave propagation speed along the corresponding line branch.

We now take the voltage wave at the observation point OP1 $x_{1}=x_{o 1}$ located between $x_{1}=0$ and the source point $x_{1}=x_{s}$ as an example ${ }^{3}$ :

$$
V_{x_{o 1}}^{\mathrm{DT}}(t)=-\frac{1}{2} V_{S}\left(t-\tau_{1}^{\prime}\right)+\rho_{2,3}^{1} \cdot \frac{1}{2} V_{S}\left(t-\tau_{1}^{\prime \prime}\right),\left(x_{o 1}<x_{s}\right),
$$

with the propagation delays respectively being

$$
\tau_{1}^{\prime}=\frac{x_{s}-x_{o 1}}{v_{1}}
$$

and

$$
\tau_{1}^{\prime \prime}=\frac{2 L_{1}-x_{s}-x_{o 1}}{v_{1}} .
$$

We denote the time-reversed copy of $V_{i}(t)$ as

$$
V_{i}^{\mathrm{tr}}(t)=V_{i}(T-t),
$$

where, to ensure causality, the time-reversal operation is given by the transformation

$$
t \Longrightarrow T-t
$$

with $T$ being the length of the time window over which the terminal voltage responses are recorded.

In the backward-propagation stage [see Fig. 3(b)], the synchronous excitation of the time-reversed sources [also named secondary sources relative to the original source $V_{S}(t)$ ] generates the voltage wave at $x_{1}=x_{o 1}$ through

$$
V_{x_{o 1}}^{\mathrm{RT}}(t)=\sum_{i=1}^{3} V_{x_{o 1}}^{\mathrm{RT}: V_{i}^{\mathrm{tr}}}(t)
$$

\footnotetext{
${ }^{3}$ The superscripts DT and RT are used to indicate, respectively, that the signals are evaluated in the forward- and backward-propagation stage (i.e., direct time and reversed time) of the closed time-reversal cavity.
} 


$$
\begin{aligned}
2 V_{x_{o} 1}^{\mathrm{RT}}(t)= & -V_{S}\left(T-t-\tau_{1}^{\prime}\right)+\rho_{2,3}^{1} \cdot V_{S}\left(T-t-\tau_{1}^{\prime \prime}\right)-\rho_{2,3}^{1} \cdot V_{S}\left(T-t+\tau_{1}^{\prime \prime}\right)+\left(\rho_{2,3}^{1}\right)^{2} \cdot V_{S}\left(T-t+\tau_{1}^{\prime}\right) \\
& +\left(1+\rho_{1,3}^{2}\right) \cdot\left(1+\rho_{2,3}^{1}\right) \cdot V_{S}\left(T-t+\tau_{1}^{\prime}\right)+\left(1+\rho_{1,2}^{3}\right) \cdot\left(1+\rho_{2,3}^{1}\right) \cdot V_{S}\left(T-t+\tau_{1}^{\prime}\right) \\
V_{x_{o} 1}^{\mathrm{RT}}(t)= & \underbrace{\rho_{2,3}^{1} \cdot \frac{1}{2} V_{S}\left(T-t-\tau_{1}^{\prime \prime}\right)-\frac{1}{2} V_{S}\left(T-t-\tau_{1}^{\prime}\right)}_{\text {Converging }} \underbrace{+\frac{1}{2} V_{S}\left(T-t+\tau_{1}^{\prime}\right)-\rho_{2,3}^{1} \cdot \frac{1}{2} V_{S}\left(T-t+\tau_{1}^{\prime \prime}\right)}_{\text {Diverging }} .
\end{aligned}
$$

where $V_{i}^{\text {tr }}$ in the superscript indicates the individual contribution from the time-reversed voltage $V_{i}^{\mathrm{tr}}(t)$ being separately back injected at its corresponding terminal. The contributions can be readily obtained as ${ }^{4}$ :

$$
\begin{gathered}
V_{x_{o 1}}^{\mathrm{RT}: V_{1}^{\mathrm{tr}}}(t)=\frac{1}{2} \cdot 2 V_{1}^{\mathrm{tr}}\left(t-\frac{x_{o 1}}{v_{1}}\right)+\rho_{2,3}^{1} \cdot \frac{1}{2} \cdot 2 V_{1}^{\mathrm{tr}}\left(t-\frac{L_{1}}{v_{1}}-\frac{L_{1}-x_{o 1}}{v_{1}}\right), \\
V_{x_{o 1}}^{\mathrm{RT}: V_{2}^{\mathrm{tr}}}(t)=\left(1+\rho_{1,3}^{2}\right) \cdot \frac{1}{2} \cdot 2 V_{2}^{\mathrm{tr}}\left(t-\frac{L_{2}}{v_{2}}-\frac{L_{1}-x_{o 1}}{v_{1}}\right), \\
V_{x_{o 1}}^{\mathrm{RT}: V_{3}^{\mathrm{tr}}}(t)=\left(1+\rho_{1,2}^{3}\right) \cdot \frac{1}{2} \cdot 2 V_{3}^{\mathrm{tr}}\left(t-\frac{L_{3}}{v_{3}}-\frac{L_{1}-x_{o 1}}{v_{1}}\right) .
\end{gathered}
$$

Substituting (12) - (14) into (11) yields (15).

According to the boundary condition at the junction,

$$
\rho_{2,3}^{1}+\rho_{1,3}^{2}+\rho_{1,2}^{3}=-1
$$

Using (16), $V_{x_{o 1}}^{\mathrm{RT}}(t)$ is simplified as (17).

Following a similar approach, we can derive the solutions for the voltage waves observed along Line branch 2 (i.e., at $\left.x_{2}=x_{o 2}\right)$ in the forward- and backward-stages:

$$
V_{x_{o 2}}^{\mathrm{DT}}(t)=\left(1+\rho_{2,3}^{1}\right) \cdot \frac{1}{2} V_{S}\left(t-\tau_{2}\right),
$$

with

and

$$
\tau_{2}=\frac{L_{1}-x_{s}}{v_{1}}+\frac{x_{o 2}}{v_{2}}
$$

$$
\begin{aligned}
& V_{x_{o 2}}^{\mathrm{RT}}(t)= \\
& \underbrace{\left(1+\rho_{2,3}^{1}\right) \cdot \frac{1}{2} V_{S}\left(T-t-\tau_{2}\right)}_{\text {Converging }} \underbrace{-\left(1+\rho_{2,3}^{1}\right) \cdot \frac{1}{2} V_{S}\left(T-t+\tau_{2}\right)}_{\text {Diverging }} .
\end{aligned}
$$

The voltage wave expression obtained at the observation point $x_{3}=x_{o 3}$ along Line branch 3 has a similar form as that at $x_{2}=x_{o 2}$ :

$$
\begin{gathered}
V_{x_{o 3}}^{\mathrm{DT}}(t)=\left(1+\rho_{2,3}^{1}\right) \cdot \frac{1}{2} V_{S}\left(t-\tau_{3}\right), \\
V_{x_{o 3}}^{\mathrm{RT}}(t)= \\
\underbrace{\left(1+\rho_{2,3}^{1}\right) \cdot \frac{1}{2} V_{S}\left(T-t-\tau_{3}\right)}_{\text {Converging }} \underbrace{-\left(1+\rho_{2,3}^{1}\right) \cdot \frac{1}{2} V_{S}\left(T-t+\tau_{3}\right)}_{\text {Diverging }},
\end{gathered}
$$

\footnotetext{
${ }^{4}$ When $V_{i}^{\mathrm{tr}}(t)$ is back injected as the secondary source, a voltage division ratio of $1 / 2$ caused by the matched boundary condition at the network terminal is introduced. As seen in (12) - (14), $V_{i}^{\mathrm{tr}}(t)$ is multiplied by 2 to offset the dividing effect, to be consistent with the generic definition of the closed timereversal cavity.
}

where

$$
\tau_{3}=\frac{L_{1}-x_{s}}{v_{1}}+\frac{x_{o 3}}{v_{3}} .
$$

We now use the expressions (21) and (22) of $x_{3}=x_{o 3}$ as an example to interpret the properties of the voltage wave propagation in the forward- and backward-propagation stages.

Basically, the voltage wave observed in the backward stage consists of two components, which arrive at the observation point one after the other. The later-arriving component [i.e., the second term in (22)] is an inverted and time-delayed copy of the first wave. The two components are respectively generated by the processes of the time-reversed terminal responses (back injected by the secondary sources) converging to and diverging from the original source location. For this reason, the two components are referred to, respectively, as the 'converging' and 'diverging' terms in (22), in agreement with the terminology used in [1]-[3].

It is important to note that only the converging wave in the backward stage behaves as a time-reversed copy of the voltage wave in the forward stage, whereas the diverging component does not have any counterpart in the forward stage. This can be clearly seen by comparing equations (17), (20) and (22) with their forward-stage counterparts, namely equations (6), (18) and (21), respectively. In other words, the earlier-arriving converging component is a mirror image of the voltage wave observed in the forward stage with respect to the recording time-window length $T$.

We now discuss the interfering effect ${ }^{5}$ caused by the diverging wave for observation points located in the region near the source location.

For ease of explanation, the components of $V_{x_{o 1}}^{\mathrm{RT}}$ in (17) are arranged in chronological order according to the instants when they arrive at the observation point $x_{1}=x_{o 1}$. It can be seen that the difference (denoted by $\tau$ ) between the instants of the converging and diverging components respectively arriving at $x_{1}=x_{o 1}$ is twice the prorogation delay $\tau_{1}^{\prime}$. An immediate consequence of this fact is that the diverging voltage wave overlaps the converging one at the region near the source location that is characterized by a relatively small $\tau_{1}^{\prime}$. The two voltage waves can be completely separated in time if $\tau$ is greater than the temporal duration $T_{S}$ of the voltage source $V_{S}(t)$. Thus, this interfering would affect only the observation point that is located around the source and is characterized by the abscissa $x_{1}=x_{o 1}$ satisfying

$$
\left|x_{o 1}-x_{s}\right| \leqslant \frac{1}{2} v_{1} \cdot T_{S}
$$

\footnotetext{
${ }^{5}$ Note that Fink and his co-workers used the term 'distortion' in their papers (e.g., [1]-[3]). We preferred to use here instead the term interfering to avoid any confusion with the concept of distortion in communication systems.
} 
Here, without loss of generality, we assume that Line branch 1 is long enough so that the interfering region is confined along the line branch where the source excitation is originally injected.

This conclusion is also analogous to that of the time-reversal cavity for acoustic waves in free space [1]-[3].

In summary, the closed time-reversal cavity is realized for the observation points that satisfy the following condition

$$
V^{\mathrm{RT}, c} \cdot(x, t)=V^{\mathrm{DT}}(x, T-t), \quad\left(x>x_{s} \pm \frac{1}{2} v_{1} \cdot T_{S}\right)
$$

where $V^{\mathrm{RT}, c \cdot}(x, t)$ represents the converging component of the voltage wave at a generic location $x$ along the network.

\section{NUMERICAL AND EXPERIMENTAL VALIDATION}

The preceding analysis demonstrated the realization of a closed time-reversal cavity for electromagnetic waves in transmission line networks. It was shown that the voltage wave resulting from the converging process in the backward stage is a time-reversed copy of the voltage wave that was originally generated by the source in the forward stage. This section presents numerical and experimental validation of the proposed closed time-reversal cavity. To this end, the generic Y-shaped inhomogeneous cable network of Fig. 3 is experimentally realized using the setup described in Fig. 4.
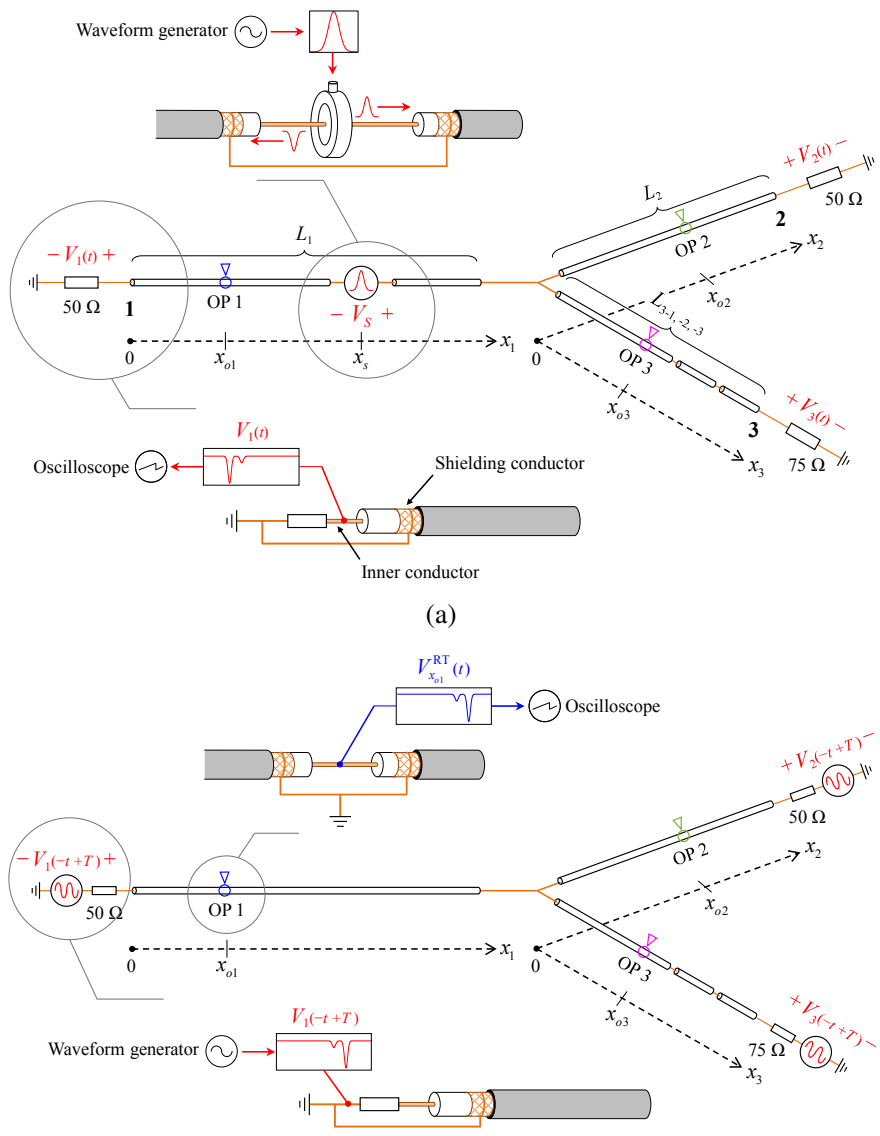

(b)

Fig. 4: Experimental setups realizing a time-reversal cavity in a Y-shaped transmission line network. (a) Forward-propagation stage: direct time. (b) Backward-propagation stage: reversed time.
TABLE I: Configuration of the setup described in Fig. 4

\begin{tabular}{c|c|c|c}
\hline Line branch & Cable & \multicolumn{2}{|c}{ Length } \\
\hline 1 (along the $x_{1}$ axis) & RG-58 & $L_{1}$ & $45 \mathrm{~m}$ \\
\hline 2 (along the $x_{2}$ axis) & RG-58 & $L_{2}$ & $26 \mathrm{~m}$ \\
\hline \multirow{3}{*}{3 (along the $x_{3}$ axis) } & RG-59 & $L_{3-1}$ & $14 \mathrm{~m}$ \\
\cline { 2 - 4 } & RG-179 & $L_{3-2}$ & $4 \mathrm{~m}$ \\
\cline { 2 - 4 } & RG-59 & $L_{3-3}$ & $4 \mathrm{~m}$ \\
\hline
\end{tabular}

TABLE II: Main parameters of RG-58/59/179 coaxial cable

\begin{tabular}{c|c|c|c}
\hline & RG-58 & RG-59 & RG-179 \\
\hline Characteristic impedance $(\Omega)$ & 50 & 75 & 75 \\
\hline $\begin{array}{c}\text { Propagation speed } \\
\text { (\% of the speed of light) }\end{array}$ & 66 & 65.8 & 69 \\
\hline $\begin{array}{c}\text { Nom. attenuation }(\mathrm{dB} / \mathrm{m}) \\
(f \leq 35 \mathrm{MHz})\end{array}$ & 0.07 & 0.06 & 0.14 \\
\hline $\begin{array}{c}\text { Diameter of inner/shielding } \\
\text { conductor }(\mathrm{mm} / \mathrm{mm})\end{array}$ & $0.9 / 3.6$ & $0.58 / 4.5$ & $0.31 / 2$ \\
\hline
\end{tabular}

The proposed network is composed of three line branches and five segments of coaxial cables. As detailed in Table I, Line branches 1 and 2 are RG-58 coaxial cables, which are respectively $45 \mathrm{~m}$ and $26 \mathrm{~m}$ in length. Note that Line branch 3 contains a mix of three line sections comprising RG-59 and RG-179 coaxial cables. The main parameters of the adopted RG-58, -59 and -179 standard coaxial cables are summarized in Table II. The three types of cables feature different characteristic impedances, wave propagation speeds and cross-sectional dimensions. As a result, the considered cable network is characterized by a high degree of inhomogeneity.

Matching the coaxial-cable network under study consists of terminating RG-58 and -59 cables with lumped resistors of 50 $\Omega$ and $75 \Omega$, respectively. Note that matched impedances are only required at the external terminations.

Figure 5 illustrates the profile of a modified Gaussian pulse that was adopted as the source signal of the series voltage source $V_{S}(t)$ to excite the network in the forward-propagation stage. The $1 / e$ pulse width of the injected Gaussian pulse is $38 \mathrm{~ns}$. In this respect, the width is sufficiently small in the light of the propagation delays of the line branches.

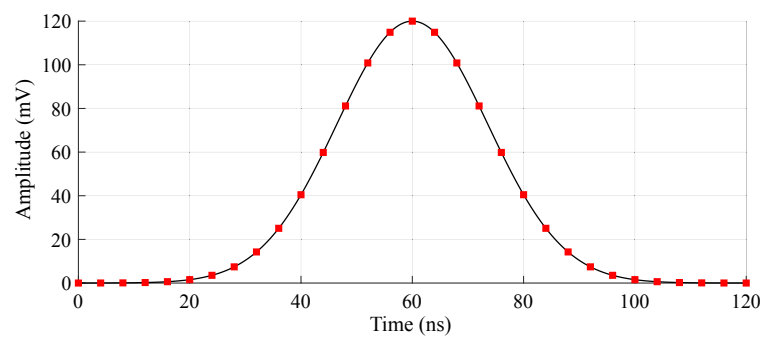

Fig. 5: Modified Gaussian pulse as the excitation signal of the series voltage source $V_{S}(t)$. The red squares show the sampling points which correspond to the $250 \mathrm{MS} / \mathrm{s}$ sampling rate of the adopted arbitrary waveform generator.

\section{A. Numerical validation}

The voltage wave propagation along the cable network representing a closed time-reversal cavity was first numerically simulated within the EMTP-RV environment [22], [23]. In the numerical simulations, losses in the coaxial cables were 
disregarded as in the foregoing derivation. This way, the timereversal invariance of the underlying telegrapher's equations is strictly satisfied.

In accordance with the setup described in Fig. 4(a), Fig. 6 presents the terminal voltages $V_{i}(t)$ ( $i$ being 1 to 3 ) simulated in the forward-propagation stage of the time-reversal cavity. The series voltage source $V_{S}(t)$ injected the Gaussian pulse at $x_{1}=x_{s}=28 \mathrm{~m}$, giving rise to the voltage wave propagating toward the network terminals. The voltage responses at the terminals were observed in a time window $T$ of $800 \mathrm{~ns}$. The voltages $V_{i}(t)$ were then time reversed and shifted in time by $T$ as $V_{i}(T-t)$. The two sets of waveforms are superimposed in Fig. 6. The original waveforms $V_{i}(t)$ and their time-reversed images $V_{i}(T-t)$ are symmetrical with respect to the middle point of the time window $T$ (i.e., $t=400 \mathrm{~ns}$ ).

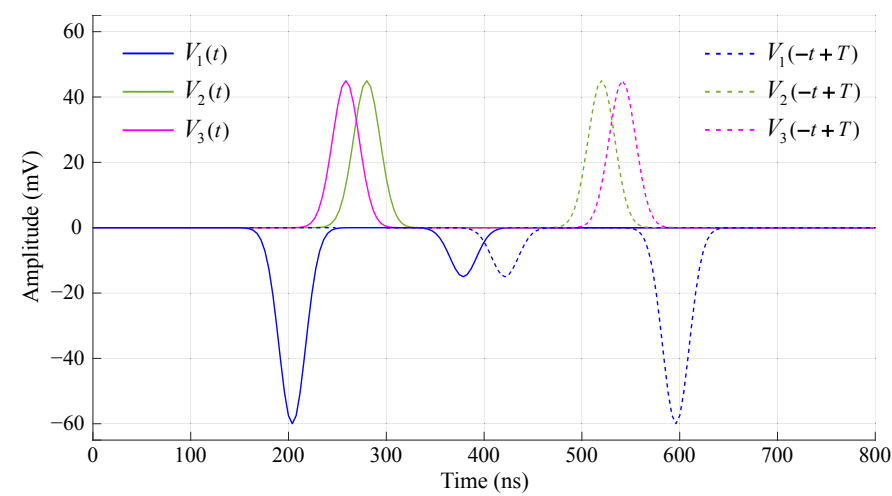

Fig. 6: Simulated terminal voltage waveforms $V_{i}(t)$ in the forwardpropagation stage of the closed time-reversal cavity. The terminal responses are time reversed as $V_{i}(T-t)$ and plotted in dashed lines.

For simulating the backward propagation, the time-reversed voltages $V_{i}(T-t)$ were synchronously back injected into the network. In view of the line parameters reported in Tables I and II, the interfering effect caused by the diverging process in the Y-shaped cable network can be eliminated by considering the observation points with a coordinate satisfying either $x_{1}$ $\leq 16 \mathrm{~m}$ or $x_{1} \geq 40 \mathrm{~m}$. Fig. 7 presents the simulated voltage waves in the forward-propagation stage (solid lines) and the backward-propagation stage (dashed lines) at the three observation points, which are located along the three line branches with the coordinates $x_{1}=x_{o 1}=4 \mathrm{~m}, x_{2}=x_{o 2}=17$ $\mathrm{m}$ and $x_{3}=x_{o 3}=12 \mathrm{~m}$, respectively. As analyzed previously, the observed voltage waves contributed by the converging process in the backward stage are exact time-reversed copies of the voltage waves propagating in the forward stage.

\section{B. Experimental validation}

The proposed Y-shaped coaxial-cable network realizing a closed time-reversal cavity was validated experimentally. The expanded views included in Fig. 4 describe the involved experimental devices and approaches. The main specifications of the devices used in the experiment are summarized in Table III. Note that the spectrum of the Gaussian pulse of Fig. 5 extends to significant frequencies of up to ten $\mathrm{MHz}$.

In the forward-propagation stage, an arbitrary waveform generator was used in combination with a Pearson 1 V/A (50

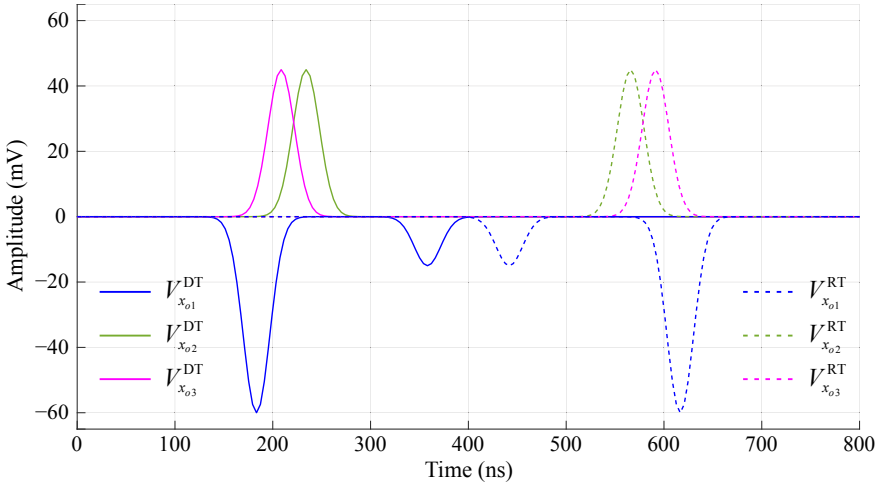

Fig. 7: Simulated voltage waveforms at the considered observation points. Solid lines: forward-stage voltages. Dashed lines: backwardstage voltages.

TABLE III: Main characteristics of the experimental devices

\begin{tabular}{c|c|c|c}
\hline & $\mathrm{CP}^{a}$ & $\mathrm{AWG}^{b}$ & Osc. $^{c}$ \\
\hline Bandwidth & $200 \mathrm{MHz}$ & $125 \mathrm{MHz}$ & $350 \mathrm{MHz}$ \\
\hline Sampling rate & - & $250 \mathrm{MS} / \mathrm{s}$ & $2.5 \mathrm{GS} / \mathrm{s}$ \\
\hline Vertical resolution & - & $16 \mathrm{bit}$ & $14 \mathrm{bit}$ \\
\hline
\end{tabular}

turns) broadband current transformer for the generation and injection of a voltage pulse into the inner conductor of the coaxial cable [see Fig 4(a)]. Corresponding to the simulation scenario, the current probe was positioned along Line branch 1 at $x_{1}=x_{s}=28 \mathrm{~m}$. With the injection of a signal from the secondary coil, the current probe behaves as a voltage transformer with a transformation ratio of 50:1. Therefore, the same Gaussian pulse shown in Fig. 5 was applied to the secondary coil of the current probe but with a peak value of 6 $\mathrm{V}$. This way, the generated voltage pulse, which is equivalent to the excitation of the series voltage source $V_{S}(t)$ in the inner conductor in Fig. 4(a), has a peak value of $120 \mathrm{mV}$. At the matched line terminals, the induced voltage responses were recorded by means of an oscilloscope.

The measured terminal voltages are shown in Fig. 8. In the same figure, the simulated counterparts are also presented in black lines for the sake of comparison. It can be seen that, except for the peak values that are attenuated in the measured signals, the overall simulated and measured waveforms are in high-grade agreement. The attenuation is essentially due to the losses in the cables, which were not considered in the simulations.

As described in Fig. 4(b), in the backward stage, the responses acquired in the forward stage at the network terminals were reversed in time and synchronously back injected into the network from the respective terminals using an arbitrary waveform generator.

Figure 9 presents the voltages measured in the forward stage (solid lines) and in the backward stage (dashed lines) at the three considered observation points. It is evident that the observed signals in the backward stage (more precisely of the converging process) are nearly-perfect time-reversed copies of the ones originally present in the forward stage. The difference between the two stages' voltages is the slight attenuation in amplitude, which stems from losses in the cables. Note that 


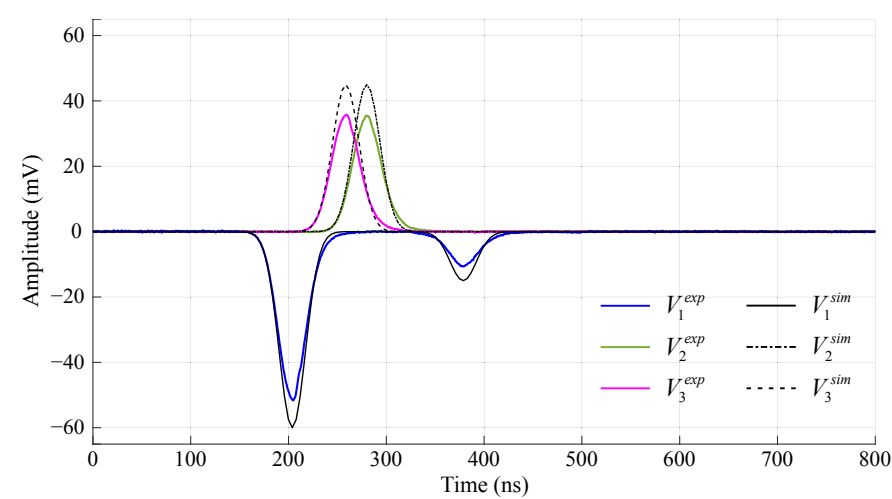

Fig. 8: Experimentally recorded terminal voltage waveforms $V_{i}(t)$ (indicated by the superscript exp in the legend) in the forward stage. The simulated waveforms (indicated by the superscript sim) are shown in black (solid for $V_{1}$, dash-dotted for $V_{2}$ and dashed for $V_{3}$ ).

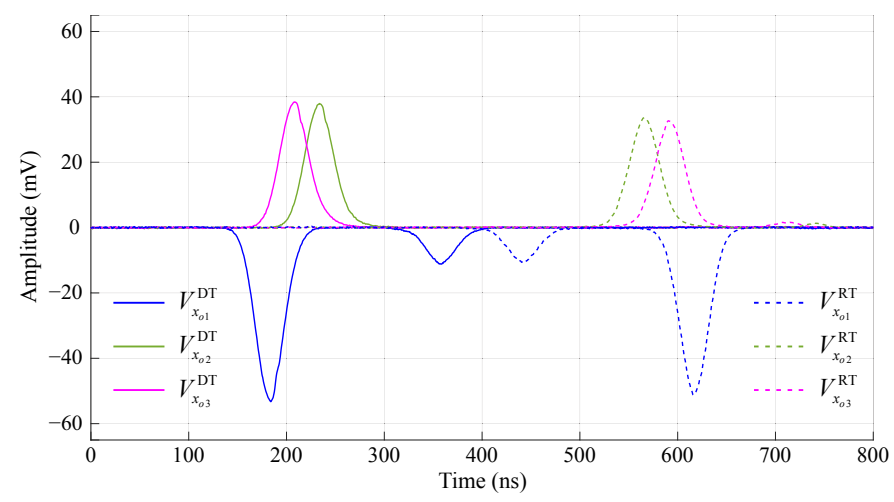

Fig. 9: Experimentally recorded voltage waveforms at the considered observation points. Solid lines: forward-stage voltages. Dashed lines: backward-stage voltages.

by considering an inverted-loss medium in the backward stage [24], the attenuation effect can be compensated.

\section{Discussion}

The preceding results have validated the wave propagation property of the closed time-reversal cavity realized by the Yshaped cable network. The observation refers to the locations that are out of the interfering region. The discussion in this subsection focuses on the locations neighboring the original source location $x_{1}=x_{s}$.

\section{A. Interfering effect of diverging voltage waves}

Given the previous analysis, the interfering region for the proposed time-reversal cavity is bounded at $x_{1}=x_{s} \pm 12 \mathrm{~m}$. Figs. 10(a) and (b) depict the voltage waves measured at the locations $x_{1}=x_{s} \pm \Delta x$ with $\Delta x$ being 1,2 and $5 \mathrm{~m}$, respectively. As formulated previously, if the observation point is within the interfering region, the backward-stage voltage wave is not any more a time-reversed copy of its counterpart in the forward stage.

The forward-stage voltage wave can be decomposed into two terms (see the waveforms of solid lines in Fig. 10): $i$ ) the incident voltage wave generated by the source denoted by the superscript ' $i$ ' and $i i$ ) the reflected voltage wave from the junction point denoted by the superscript ' $r$ '. The incident voltage wave has the same shape as the series excitation source

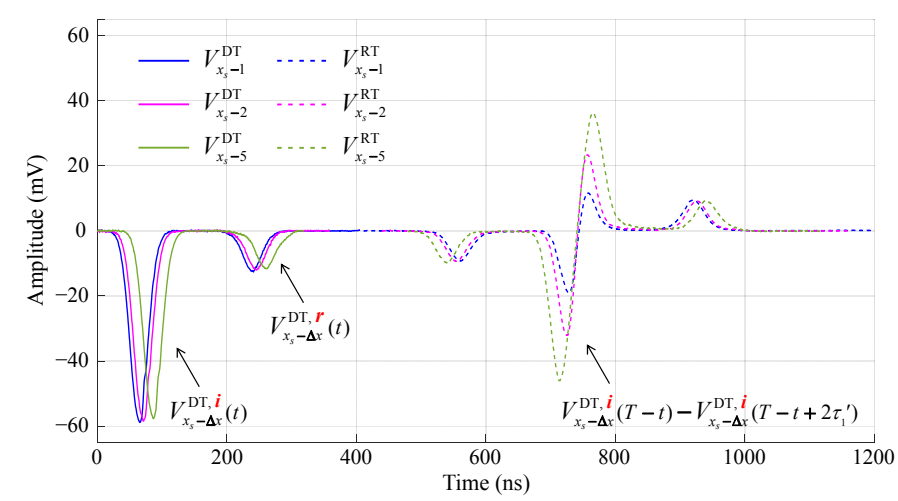

(a)

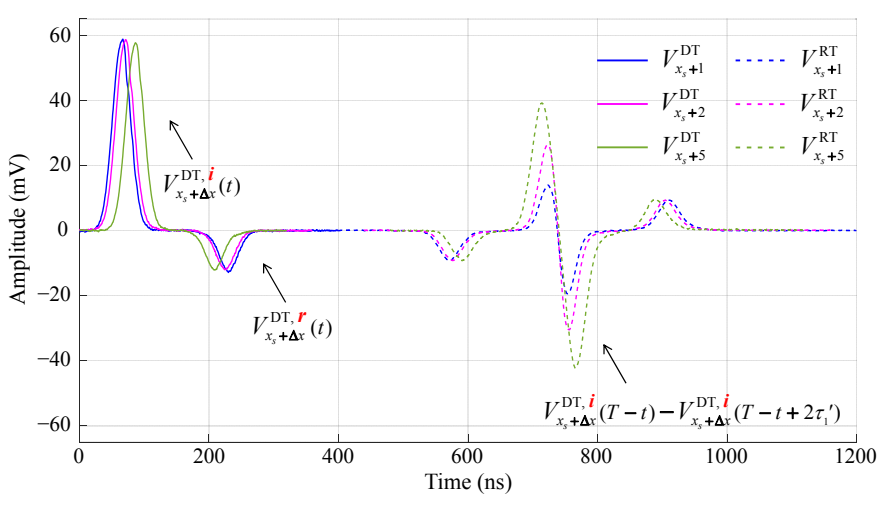

(b)

Fig. 10: Experimentally recorded voltage waveforms at the points neighboring the source location $x_{1}=x_{s}$. Solid lines: voltages in the forward stage. Dashed lines: voltages in the backward stage. The observation points are on (a) the side $x_{1}<x_{s}$ and (b) the side $x_{1}>x_{s}$.

$V_{S}(t)$ but with an amplitude reduced by a factor 2 due to the division of the excitation voltage. As can be seen in Figs. 10(a) and 10(b), the incident voltage wave has either a positive or negative polarity depending on whether the observation point is located on the right side (i.e., $x_{1}>x_{s}$ ) or on the left side (i.e., $x_{1}<x_{s}$ ) of the series voltage source.

In the backward-propagation stage, the interfering effect results from the time-reversed incident waves $V_{x_{s} \pm \Delta x}^{\mathrm{DT}, i}(T-t)$, namely the converging waves, overlapping with their respective inverted and time-shifted copies (i.e., the diverging waves). It is also demonstrated by the results that only the incident component that is originally generated by the source itself is distorted by the diverging voltage wave in the backward stage.

It is worth noting that in spite of the overlap between the converging and diverging voltage waves, it is still possible to locate the position of the source. Thanks to the timereversal temporal-spatial correlation property, $V_{x_{s}}^{\mathrm{DT}, i}(T-t)$ and its inverted copy inherently arrive in phase at the source location $x_{1}=x_{s}$, resulting in a complete offset. This way, the original source point can be exclusively distinguished from its neighboring locations, for example, by means of an algorithm assessing the energy of the voltage wave along the network.

Figure 11 shows the normalized voltage energy as a function of the distance to the network terminal $x_{1}=0$. Obviously, the source location $\left(x_{1}=28 \mathrm{~m}\right)$ is characterized by the global minimum voltage energy among the observation points 
distributed along the network.

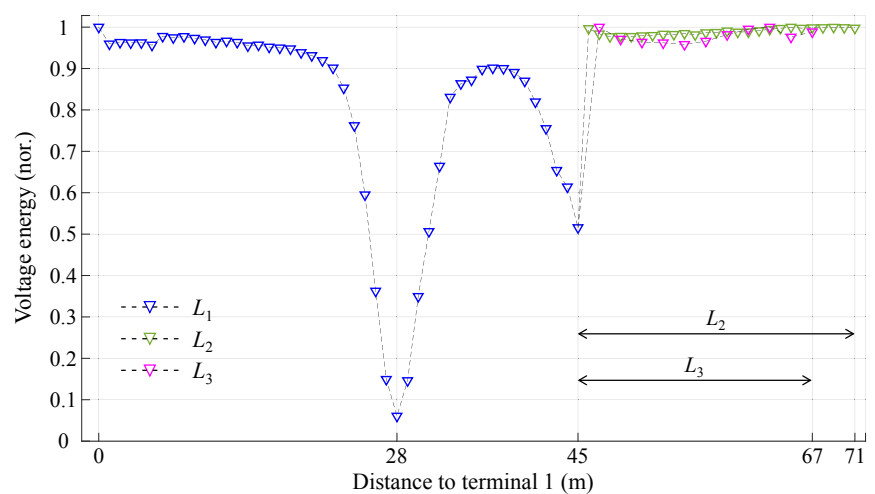

Fig. 11: Normalized energy of the voltage experimentally recorded along the network. The spacings of the observation points are, respectively, $1 \mathrm{~m}$ along Line branches 1 and 2, and $2 \mathrm{~m}$ along Line branch 3 . The normalization is based on the maximal voltage energies calculated for the respective line branches.

\section{B. A closed time-reversal cavity in transmission line networks} with a time-reversal sink

Rosny and Fink have proposed in acoustics the concept of a time-reversal sink to overcome the interfering effect caused by the diverging waves in the region near the source location [25]. According to this concept, during the backward-propagation stage of the time-reversal process, the source is replaced by a sink that absorbs the incident wave.

Both active and passive realizations of a time-reversal sink are possible. For the case of a single transmission line, a passive time-reversal sink can be straightforwardly designed as a simple two-port passive circuit at the source location. However, when it comes to a topologically-complex transmission line network (characterized by multiple junctions and terminals), the realization of a passive sink would be quite challenging and it would require dedicated time-varied switch operations.

However, if the source excitation is known, the sink can be experimentally realized by an active source that generates an additive inverse of the diverging wave in the backward stage, compensating therefore the interfering effect caused by the diverging process.

Assume that the original source remains active in the backward-propagation stage but with a time-reversed waveform, namely $V_{S}(T-t)$ for the proposed case of the time-reversal cavity in transmission line networks. Mathematically, solving the wave equation taking into account the active time-reversal sink in the backward stage, it can readily be shown that the voltage wave generated by the time-reversed source is identical to the diverging term in (17) but with opposite polarity.

Figure 12 compares the distorted voltage wave experimentally recorded at the location $x_{1}=x_{s}-1$ as an example with the one measured in presence of the active source $V_{S}(T-t)$ representing a time-reversal sink. As can be observed, even the voltage wave is recorded at a location quite adjacent to the source point, its waveform in the backward-propagation stage is nearly a time-reversed copy of the voltage wave in the forward-propagation stage, as the diverging voltage wave has been compensated.

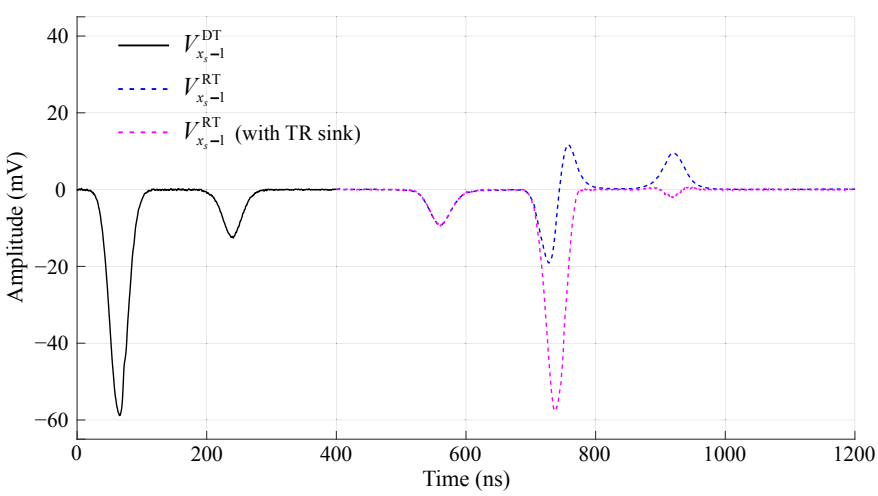

Fig. 12: Experimentally-recorded voltage waveforms at the location $x_{1}=x_{s}-1$ in the forward-propagation stage (i.e., with the black solid line) and the backward-propagation stage [with and without a time-reversal (TR) sink].

Thus, the so-called time-reversal sink applies the additional source generated wave to interfere destructively with the backward-stage diverging wave, keeping intact the converging component.

\section{CONCLUSIONS}

We demonstrated that a closed time-reversal cavity can be realized using a transmission line network. Such a timereversal cavity requires a finite number of observation points located at the terminals of the network. A time-reversed copy of the system state (e.g., voltage wave distributions) originated by a source in the forward-propagation stage can be obtained by time-reversing the response at the terminals and back injecting them into the network.

In the experimental realization of the time-reversal cavity, the resulting distribution was affected by an attenuation resulting from losses in the coaxial cables. Indeed, the wave equations in transmission lines are time-reversal invariant for lossless lines. When the network is lossy, the time-reversal invariance will hold when an inverted-loss medium is considered in the back-propagation model [24]. An inverted-loss medium can be considered in a straightforward way in numerical simulations as long as the line losses are well characterized.

We showed in this study that the past behavior of signals along a transmission line network can be reproduced in the future. To achieve this, only a finite number of responses need to be monitored at the network terminals. We also discussed the interfering effect of the so-called diverging wave for observation points in the vicinity of the source. We proposed an active realization of a time-reversal sink, using the timereversed source generated wave to interfere destructively with the backward-stage diverging wave.

The proposed closed time-reversal cavity in transmission lines can find useful applications, for instance in online monitoring of communication networks, which work generally under matched conditions. Such networks can also include point-to-point wireless communication systems, which can be represented by their equivalent models in the backward stage. The synchronized measurement of signals at each network terminal would allow detecting anomalies and locating their source. The technique can also be used in various other 
applications such as: $i$ ) preventive maintenance and monitoring of cables to detect and locate incipient faults, before their occurrence; ii) detecting and locating intentional electromagnetic interference (IEMI) attacks; iii) facilitating the investigation of incidents in flight data recorders.

\section{REFERENCES}

[1] D. Cassereau and M. Fink, "Time-reversal of ultrasonic fields. iii. theory of the closed time-reversal cavity," IEEE Trans. Ultrason., Ferroelec., Freq. Contr., vol. 39, pp. 579-592, Sep. 1992.

[2] D. Cassereau and M. Fink, "Theoretical modelisation of time-reversal cavities, application to self-focussing in inhomogeneous media," Acoustical Imaging, vol. 19, pp. 141-147, 1992.

[3] D. Cassereau, F. Wu, and M. Fink, "Limits of self-focusing using closed time-reversal cavities and mirrors-theory and experiment," in IEEE Symposium on Ultrasonics, pp. 1613-1618 vol.3, Dec 1990.

[4] R. Carminati, R. Pierrat, J. de Rosny, and M. Fink, "Theory of the time reversal cavity for electromagnetic fields," Opt. Lett., vol. 32, pp. 31073109, Nov 2007.

[5] M. Fink, C. Prada, F. Wu, and D. Cassereau, "Self focusing in inhomogeneous media with time reversal acoustic mirrors," in Proceedings., IEEE Ultrasonics Symposium, pp. 681-686 vol.2, Oct 1989.

[6] C. Prada, F. Wu, and M. Fink, "The iterative time reversal mirror: A solution to self-focusing in the pulse echo mode," The Journal of the Acoustical Society of America, vol. 90, no. 2, pp. 1119-1129, 1991.

[7] M. Fink, "Time reversal of ultrasonic fields. i. basic principles," IEEE Trans. Ultrason., Ferroelec., Freq. Contr., vol. 39, pp. 555-566, Sep. 1992.

[8] J. de Rosny, G. Lerosey, and M. Fink, "Theory of electromagnetic timereversal mirrors," IEEE Trans. Antennas Propagat., vol. 58, pp. 31393149 , Oct 2010.

[9] F. Rachidi, M. Rubinstein, and M. Paolone, Electromagnetic Time Reversal: Application to EMC and Power Systems. John Wiley \& Sons, 2017.

[10] L. Borcea, G. Papanicolaou, C. Tsogka, and J. Berryman, "Imaging and time reversal in random media," Inverse Problems, vol. 18, pp. 12471279, Oct. 2002.

[11] D. Liu, G. Kang, L. Li, Y. Chen, S. Vasudevan, W. Joines, Q. Liu, J. Krolik, and L. Carin, "Electromagnetic time-reversal imaging of a target in a cluttered environment," IEEE Trans. Antennas Propagat., vol. 53, pp. 3058-3066, Sept. 2005.

[12] Q. Gao, B. Wang, and X. Wang, "Far-field super-resolution imaging with compact and multifrequency planar resonant lens based on time reversal," IEEE Trans. Antennas Propagat., vol. 63, no. 12, pp. 55865592, 2015.

[13] V. C. Odedo, M. E. Yavuz, F. Costen, R. Himeno, and H. Yokota, “Time reversal technique based on spatiotemporal windows for through the wall imaging," IEEE Trans. Antennas Propagat., vol. 65, pp. 3065-3072, June 2017.

[14] S. Mukherjee, L. Udpa, S. Udpa, and E. J. Rothwell, "Target localization using microwave time-reversal mirror in reflection mode," IEEE Trans. Antennas Propagat., vol. 65, no. 2, pp. 820-828, 2017.

[15] R. Razzaghi, G. Lugrin, H. Manesh, C. Romero, M. Paolone, and F. Rachidi, "An efficient method based on the electromagnetic time reversal to locate faults in power networks," IEEE Trans. Power Deli., vol. 28, pp. 1663-1673, July 2013.

[16] A. Codino, Z. Wang, R. Razzaghi, M. Paolone, and F. Rachidi, "An alternative method for locating faults in transmission line networks based on time reversal," IEEE Transactions on Electromagnetic Compatibility, vol. 59, no. 5, pp. 1601-1612, 2017.

[17] Z. Wang, R. Razzaghi, M. Paolone, and F. Rachidi, "Electromagnetic time reversal similarity characteristics and its application to locating faults in power networks," IEEE Transactions on Power Delivery, vol. 35 , no. 4 , pp. 1735-1748, 2020.

[18] C. Draeger, J.-C. Aime, and M. Fink, "One-channel time-reversal in chaotic cavities: Experimental results,” J. Acoust. Soc. Am., vol. 105, no. 2, pp. 618-625, 1999.

[19] G. Lerosey, J. de Rosny, A. Tourin, A. Derode, G. Montaldo, and M. Fink, "Time reversal of electromagnetic waves," Phys. Rev. Lett., vol. 92, p. 193904, May 2004.

[20] A. Przadka, S. Feat, P. Petitjeans, V. Pagneux, A. Maurel, and M. Fink, "Time reversal of water waves," Phys. Rev. Lett., vol. 109, p. 064501, Aug 2012.
[21] F. Tesche, M. Ianoz, and T. Karlsson, EMC Analysis Methods and Computational Models. A Wiley-Interscience publication, Wiley, 1996.

[22] H. W. Dommel, "Digital computer solution of electromagnetic transients in single-and multiphase networks," Trans. Power App. Syst., April 1969.

[23] J. Mahseredjian, S. Dennetière, L. Dubé, B. Khodabakhchian, and L. Gérin-Lajoie, "On a new approach for the simulation of transients in power systems," Electr. Pow. Syst. Res., vol. 77, no. 11, pp. 1514-1520, 2007.

[24] R. Razzaghi, G. Lugrin, F. Rachidi, and M. Paolone, "Assessment of the influence of losses on the performance of the electromagnetic time reversal fault location method," IEEE Trans. Power Deli., vol. 32, pp. 2303-2312, Oct 2017.

[25] J. de Rosny and M. Fink, "Overcoming the diffraction limit in wave physics using a time-reversal mirror and a novel acoustic sink," Phys. Rev. Lett., vol. 89, p. 124301, Aug 2002. 